Casos Clínicos

Arch. Esp. Urol. 2010; 63 (4): 305-308

\title{
TRATAMIENTO ENDOSCÓPICO DE UN PÓLIPO FIBROEPITELIAL GIGANTE DE URETER
}

Almudena Coloma del Peso, Gloria Bocardo Fajardo, Fernando Teba del Pino, Inmaculada Fernández González, Ricardo Brime Menéndez, Atanasio Fernández Borrell, Lorenzo Herrero Torres e Ignacio Pereira Sanz.

Servicio de Urología. Hospital Universitario de La Princesa. Madrid. España.

Resumen.- OBJETIVOS: Los pólipos fibroepiteliales son tumores mesenquimales de carácter benigno, con una morfología y presentación clínica muy similar a los carcinomas uroteliales, por lo que resulta de gran importancia su diagnóstico diferencial.

METODOS/RESULTADOS: Presentamos el caso de una paciente de 42 años, con antecedentes de Linfoma Hodking mediastínico, que acude a consulta por dolor cólico lumbar derecho y hematuria. En TAC se objetiva neoforma-

\section{CORRESPONDENCIA}

Almudena Coloma del Peso Servicio de Urología Hospital Universitario de La Princesa Diego de León, 62 28006 Madrid (España).

almudenacoloma@hotmail.com Aceptado para publicar: 3 de noviembre 2009 
ción pediculada de unos $10 \mathrm{~cm}$ que protruye en vejiga con citología de orina negativa. Ante los hallazgos, y con la sospecha de pólipo fibroepitelial, se decide exploración endoscópica, que confirma el diagnóstico, y se procede a su exéresis con láser de Holmium.

DISCUSIÓN: El diagnóstico diferencial entre pólipo fibroepitelial y carcinoma urotelial no es posible realizarlo únicamente con pruebas de imagen. Se debe establecer la sospecha e indicar una exploración endoscópica, mediante ureterorrenoscopia o nefroureteroscopia percutánea según resulte más indicado, y la toma de biopsia o el tratamiento endoscópico definitivo de la tumoración.

CONCLUSIONES: La exploración endoscópica es el procedimiento de elección actualmente tanto para la confirmación diagnóstica de esta patología, como para su tratamiento definitivo.

Palabras clave: Pólipo fibroepitelial. Ureterorrenoscopia. Uréter.

Summary.- OBJETIVES: Fibroepithelial polyps are benign mesenchymal tumors with a morphology and clinical presentation very similar to transitional cell carcinomas, so that differential diagnosis is of paramount importance.

METHODS/RESULTS: We present the case of a 42-yearold female patient, with history of mediastinal Hodgkin's lymphoma, which came to the office reporting right low back colic pain and haematuria. CT scan showed pediculated neoformation of about $10 \mathrm{~cm}$ protruding into the bladder, with negative urine cytology. In view of the findings, and with the suspicion of fibroepithelial polyp, an endoscopic exploration was performed, confirming the diagnosis, followed by exeresis with Holmium laser.

DISCUSSION: A differential diagnosis between fibroepithelial polyp and transitional cell carcinoma cannot be made with imaging tests alone. The suspicion must be established, and endoscopic exploration indicated, by means of ureterorenoscopy or percutaneous nephroureteroscopy, whatever is a better indication, with biopsy or definitive endoscopic treatment of the tumor.

CONCLUSIONS: Endoscopic exploration is currently the procedure of choice for the diagnostic confirmation of this condition and its definitive treatment.

Keywords: Fibroepithelial polyps. Ureterorenoscopy. Ureter.

\section{INTRODUCCIÓN}

Los pólipos fibroepiteliales son tumores benignos de origen mesenquimal poco frecuentes, que pueden aparecer en cualquier tramo del aparato urinario. Resulta de vital importancia el diagnóstico diferencial con los carcinomas uroteliales, cuya presentación clínica y morfología en las pruebas de imagen es similar, aunque con un comportamiento y pronóstico muy diferente. En el pasado, el abordaje terapéutico de este tipo de tumores era mediante cirugía abierta. Gracias a los avances técnicos, el tratamiento endourológico, ya sea mediante ureterorrenoscopia o nefroscopia percutánea, se ha convertido actualmente en el procedimiento de elección.

Presentamos un caso de pólipo fibroepitelial gigante de uréter medio que fue tratado mediante exéresis endoscópica completa con fibra láser.

\section{MÉTODOS Y RESULTADOS}

Mujer de 42 años, con antecedentes de linfoma de Hodgkin mediastínico hace 18 años tratado con quimioterapia, actualmente en remisión completa. Acude a consulta por dolor tipo cólico en fosa renal derecha y hematuria macroscópica. Dados los antecedentes se decide estudio con citología y TAC toracoabdominal. Las citologías fueron negativas, y la tomografía evidenció adenopatías densamente calcificadas mediastínicas en relación con su patología previa, vena renal izquierda circunaórtica como variante de la normalidad, y una irregularidad y engrosamiento de uréter distal derecho, con cortes en los que parecía evidenciarse una doble luz, no concluyente. Ante los hallazgos se programa realización de ureteroscopia diagnóstica. Bajo anestesia espinal, se realiza exploración vesical encontrándose una neoformación polipoide que sobresale por orificio ureteral derecho, de superficie lisa. Se procede a la realización de ureteroscopia rígida derecha encontrando que la neoformación, de unos $10 \mathrm{~cm}$ de longitud, tiene su origen en uréter medio, con base de inserción pediculada. El resto del uréter resulta de características normales a la exploración. Con la sospecha diagnóstica de pólipo fibroepitelial ureteral, se decide sección del pedículo con láser de holmium-YAG y extracción de toda la tumoración. Se revisa la zona fotocoagulando el lecho de implantación, y se coloca un catéter doble J derecho. El postoperatorio cursa sin incidencias, por lo que se decide alta hospitalaria al segundo día postquirúrgico. El estudio histopatológico confirma la sospecha diagnóstica de pólipo fibroepitelial. Acude para la retirada de doble J tres semanas después de la cirugía. Se realiza urografía intravenosa de control a los dos meses sin encontrarse defectos de replección ni áreas de estenosis secundarias.

\section{DISCUSIÓN}

Los tumores primitivos no epiteliales benignos representan tan sólo del 5 al 10\% de los tumores del tracto urinario, siendo el pólipo fibroepitelial el más frecuente de todos ellos. Su estructura histológica consta de un eje de tejido conjuntivo revestido por epitelio malpighiano. 
Otros tumores de origen mesenquimatoso de carácter benigno que aparecen en el tracto urinario son fibromas, leiomiomas, granulomas, endometriomas, neurofibromas, hemangiomas y linfangiomas. Los pólipos fibroepiteliales, son tumores primitivos de origen mesenquimal, en principio de carácter benigno (1), aunque ha sido publicada su degeneración maligna (2). Se desconoce su etiología, pero se han postulado como posibles desencadenantes factores uroteliales irritantes crónicos como infección, inflamación, litiasis y obstrucción, aunque también se apunta su posible origen como lesiones congénitas de lento crecimiento. El primer pólipo fibroepitelial ureteral fue descrito en 1932, y desde entonces se han publicado numerosos artículos sobre este tema (3).

Se presentan principalmente en adultos entre 30 y 40 años, con predilección por el sexo masculino en una relación $3 / 2$ (4). Pueden aparecer en cualquier tramo del tracto urinario, siendo su localización más frecuente, como en nuestro caso, el uréter $(85 \%)$, seguido por la pelvis renal $(15 \%)$, y un pequeño número que se desarrolla en la uretra o la vejiga (5). Los pólipos fibroepiteliales de la pelvis renal son más frecuentes en mujeres $(79 \%)$, con predilección por el lado derecho $(79 \%)$, mientras que los de uréter lo son en hombres, y en uréter proximal del lado izquierdo (70\%), apareciendo el 62 $\%$ en la unión pieloureteral y uréter proximal. Los pólipos de tracto urinario inferior son más comunes en niños, y su localización más frecuente es la uretra posterior (4). Suelen aparecer como pólipos únicos, aunque se han publicado casos de poliposis múltiple y bilateral (5).

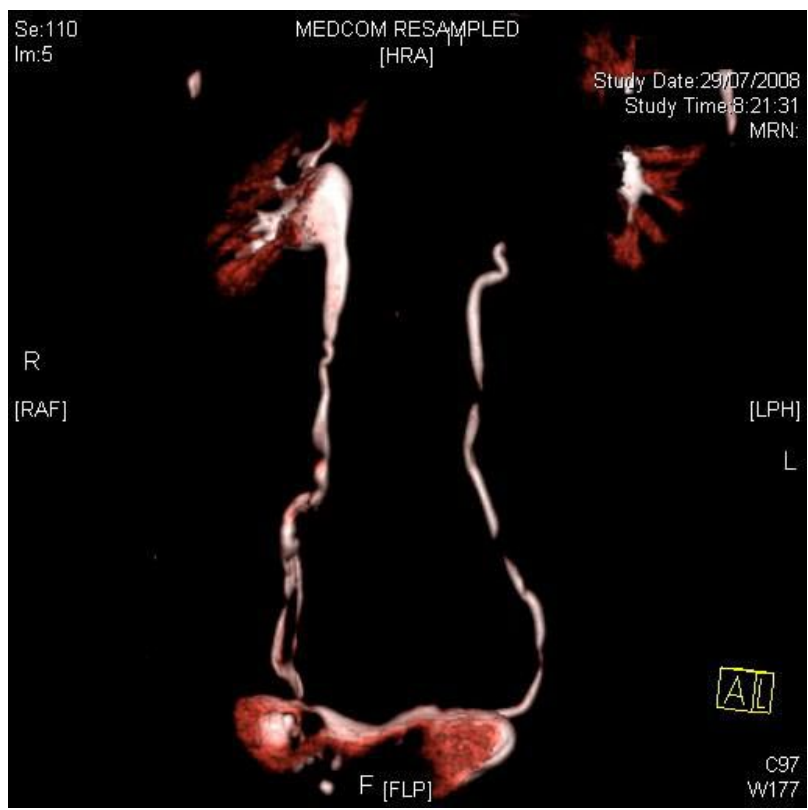

FIGURA 1. URO-TAC con reconstrucción 3D en el cual se observa el defecto de replección en tercio distal de uréter derecho con protrusión vesical.
La forma de presentación más frecuente de este tipo de tumores es dolor en flanco de características cólicas $(79 \%)$ y hematuria $(50 \%)$, clínica con la que debutó nuestra paciente, aunque también pueden aparecer otros signos y síntomas asociados menos frecuentes como disuria, polaquiuria y piuria (6), e incluso acompañados de litiasis (3).

Tanto la clínica como las pruebas de imagen resultan poco efectivas para establecer el diagnóstico diferencial definitivo entre carcinoma urotelial, de comportamiento maligno, y pólipo fibroepitelial, de carácter benigno. En nuestro caso, si no se hubiera establecido la sospecha diagnóstica previamente, no habría sido posible la confirmación diagnóstica de proceso benigno hasta después de realizado el tratamiento quirúrgico radical de un tumor de vía urinaria alta mediante nefroureterectomía.

La sospecha suele establecerse ante una imagen de defecto de replección radiolucente en la urografía intravenosa, que puede condicionar o no una ureterohidronefrosis, junto con una citología de orina negativa. Pruebas de imagen como la TAC o la cistoscopia, en el caso de pólipos fibroepiteliales con prolapso periódico en la vejiga (7), pueden ayudarnos en el intento del diagnóstico diferencial con otras patologías como coágulos, litiasis, fungusball o carcinomas uroteliales, pero la prueba diagnóstica que nos ofrecerá una mayor información en el caso de un pólipo fibroepitelial ureteral, será la exploración endoscópica de la vía urinaria superior, mediante ureterorrenoscopio rígido o flexible, que además de proporcionar una imagen directa de la tumoración, habitualmente de aspecto sésil, cilíndrico, de longitud variable y de superficie lisa, permite la toma de biopsia cuyo resultado determinará la decisión terapéutica, o el tratamiento definitivo mediante la exéresis total con posterior confirmación histológica de benignidad, como se realizó en nuestro caso.

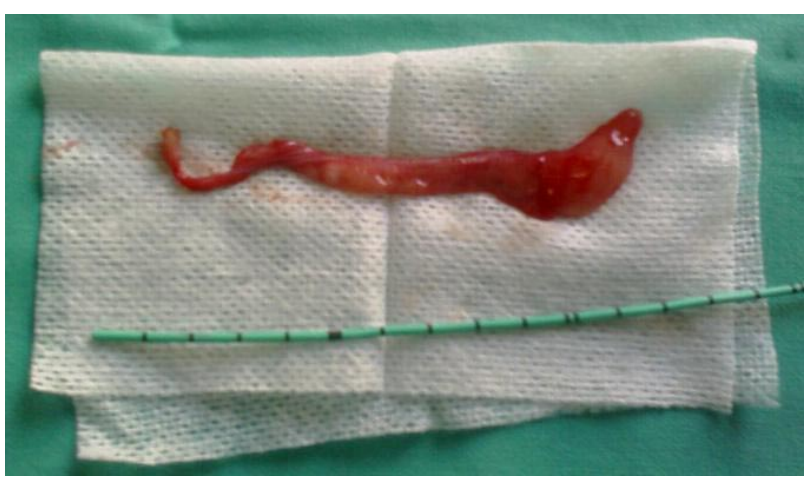

FIGURA 2. Aspecto macroscópico de la pieza una vez extraída, de aproximadamente $10 \mathrm{~cm}$ de longitud. 

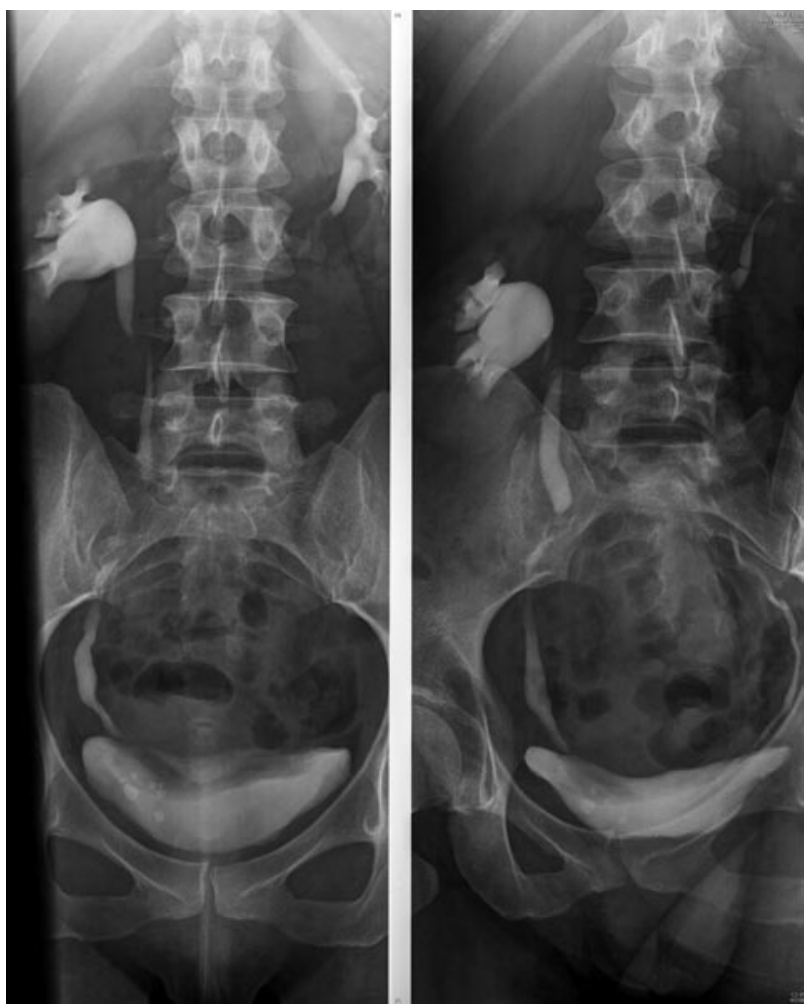

FIGURA 3. Urografía intravenosa de control 3 meses después de la cirugía, sin defectos de replección y sin estenosis secundarias al tratamiento endoscópico.

Clásicamente la resección endoscópica ha sido el gold standard en el manejo de los pólipos fibroepiteliales de pequeño tamaño, mientras que en los de gran tamaño el tratamiento más indicado resulta controvertido. En los últimos años, debido a los avances en el campo de la endourología, el tratamiento endoscópico para este tipo de tumores en uréter y pelvis renal se ha impuesto casi de manera completa. Los ureterorrenoscopios más finos y flexibles, y la aplicación de la fibra de láser de holmium para resección y coagulación de los mismos, ha permitido su resección completa mediante cirugía mínimamente invasiva (4). En nuestra paciente resultó el tratamiento de elección, ya que a pesar de su gran tamaño, fue posible el acceso endoscópico a su base de implantación, lo que permitió su exéresis completa. Se han descrito casos tratados mediante exploración y resección por cirugía abierta, 0 , incluso por laparoscopia (8), justificado por el gran tamaño de los pólipos, que, debido a su morfología sésil y su diámetro, pueden llegar a ocluir toda la luz del uréter e imposibilitar el acceso endoscópico a la base de los mismos, lo que puede resultar en una resección incompleta de la tumoración. La nefroureteroscopia anterógrada percutánea ofrece una buena alternativa para el tratamiento de estos pólipos gigantes en los que no sea posible el acceso endoscópico al pedículo de inserción, ya que permite la aproximación directa a la base del tumor, evitando su porción pediculada que crece hacia la zona más distal del uréter, lo que redunda en una adecuada visualización para la resección. Además, será el procedimiento de elección en casos de pólipos fibroepiteliales de pelvis renal, permitiendo el acceso directo a los mismos (9), y su exéresis completa.

\section{CONCLUSIÓN}

Es importante realizar el diagnóstico diferencial entre el pólipo fibroepitelial y el carcinoma urotelial de vía urinaria, ya que aunque su presentación clínica y morfología en pruebas de imagen es similar, su pronóstico y tratamiento son radicalmente distintos. La sospecha diagnóstica de pólipo fibroepitelial se debe establecer ante la constatación mediante prueba de imagen de neoformación polipoide de vía urinaria en una prueba de imagen junto con citología de orina negativa. Ante esta sospecha, actualmente, la actitud más indicada es la exploración endoscópica de la vía, con toma de biopsia para confirmación del diagnóstico, o tratamiento definitivo mediante exéresis endoscópica de la tumoración si resulta posible.

\section{BIBLIOGRAFIA y LECTURAS RECOMENDADÁS (*lectura de interés $y^{* *}$ lectura fundamental)}

1. Lovaco Castellano F, Fernández González I, García Cuerpo E, Escudero Barrilero A. Tratamiento endoscópico de los tumores del tracto urinario superior. Tratado de Urología Oncológica 2003; cap 50, Tomo II.

*2. Davides KC, King LM. Fibrous polyps of the ureter" J Urol, 1976; 115(6):651-3.

*3. Turunc T, Kuzgunbay B, Canpolat T. Ureteral fibroepithelial polyps with calculi: a case series. J Med Case Reports 2008 ; 26(2): 280.

**4. Lam JS, Bingham JB, Gupta M. Endoscopic treatment of fibroepithelial polyps of the renal pelvis and ureter. Urol, 2003; 62(5): 810-3.

5. Carey RI, Bird VG. Endoscopic management of 10 separate fibroepithelial polyps arising in a single ureter. Urol, 2006; 67(2): 413-15.

6. Williams TR, Wagner BJ, Corse WR, Vestecich JC. Fibroepithelial polyps of the urinary tract. Abdom Imaging, 2002; 27(2): 217-21.

7. Baltogiannis D, Kafetsoulis A, Giannakis D, Saito M, Miyagawa I, Sofikitis N. Fibroepithelial polyp of distal ureter with periodic prolapse into bladder. Urol Int; 81(2): 246.

**8. Kijvikai K, Maynes LJ, Herrell SD. Laparoscopic management of large ureteral fibroepithelial polyp. Urol, 2007; 70(2): 373-7.

9. Chan PH, Kho VK, Lai SK, Yang CH. Percutaneous nephroscopic resection of renal pelvic fibroepithelial polyp. J Chin Med Assoc, 2006; 69(8): 393-5. 